\title{
EFECTOS EN RATAS DE LOS ALCOHOLES DE CERA DE ABEJAS (D-002) SOBRE LA COLITIS ULCERATIVA INDUCIDA POR SULFATO DE DEXTRANO Y ETANOL
}

\author{
Vivian Molina-Cuevas ${ }^{1, a}$, Yazmin Ravelo-Calzado ${ }^{1, b}$, Zullyt Zamora-Rodríguez ${ }^{1, c}$, Miriam Noa-Puigg,d, Maikel Valle-Clara',c, \\ Yohani Pérez-Guerra ${ }^{1, b}$, Ámbar Oyarzabal-Yera ${ }^{1, b}$, Sonia Jiménez-Despaigne ${ }^{1, e}$, Rosa Mas-Ferreiro ${ }^{1, a}$
}

\begin{abstract}
RESUMEN
Objetivos. Investigar los efectos del D-002, mezcla de seis alcoholes alifáticos primarios de alto peso molecular, obtenida de la cera de abejas (Apis mellifera), sobre la colitis ulcerativa (CU) inflamatoria severa inducida por sulfato de dextrano (DSS) y etanol en ratas (Ratus ratus). Materiales y métodos. Las ratas se distribuyeron aleatoriamente en seis grupos: un control cero al que no se provocó daño, y cinco a los que se les indujo la CU: un control negativo (vehículo), tres tratados con D-002 (25, 100 y $400 \mathrm{mg} / \mathrm{kg})$ y un control positivo con sulfazalacina (200 mg/kg) (sustancia de referencia). Se cuantificaron las manifestaciones clínicas (variación del peso corporal, presencia de diarrea y de sangrado rectal), el puntaje de daño macroscópico e histológico, y la actividad de mieoloperoxidasa (MPO). Resultados. El tratamiento oral con D-002 (25, 100 y $400 \mathrm{mg} / \mathrm{kg})$ previno significativamente la disminución del peso corporal. La dosis de $400 \mathrm{mg} / \mathrm{kg}$ redujo la presencia de diarreas y sangrado rectal, aunque su comparación con el control negativo solo alcanzó significación estadística sobre las diarreas. El D-002 (25, 100 y $400 \mathrm{mg} / \mathrm{kg})$ redujo significativamente el puntaje de las lesiones macroscópicas (40,0; 43,3 y 47,2\% de inhibición, respectivamente), el puntaje de daño histológico (31,5; 53,7 y $67,1 \%$ de inhibición, respectivamente) y la actividad de MPO (73,2; 83,6 y $85,0 \%$ de inhibición, respectivamente), comparado con el grupo control negativo. La sulfazalacina redujo significativamente todas las variables estudiadas. Conclusiones. El D-002 (25, 100 y $400 \mathrm{mg} / \mathrm{kg})$ protegió significativamente la mucosa colónica en ratas con CU inflamatoria severa inducida por DSS y etanol.
\end{abstract}

Palabras clave: Abejas; Colitis ulcerosa; Ratas (Fuente: DeCS BIREME).

\section{EFFECTS IN RATS OF BEE-WAX ALCOHOLS (D-002) ON ULCERATIVE COLITIS INDUCED BY DEXTRAN SULFATE AND ETHANOL}

\begin{abstract}
Objectives. To investigate the effects of D-002, a mixture of 6 high molecular weight primary aliphatic alcohols, obtained from beeswax (Apis mellifera), on severe inflammatory ulcerative colitis (UC) induced by Dextran sulfate (DSS) and ethanol in rats (Ratus ratus). Materials and methods. Rats were randomly distributed in six groups: a zero control to which no damage was caused, and five to which the UC was induced: a negative control (vehicle), three treated with D-002 (25, 100 and $400 \mathrm{mg} / \mathrm{kg})$ and a positive control with sulfasalazine $(200 \mathrm{mg} / \mathrm{kg}$ ) (reference substance). Clinical manifestations (body weight variation, diarrhea and rectal bleeding), macroscopic and histological damage score, and myeloperoxidase (MPO) activity were quantified. Results. The oral treatment with D-002 (25, 100 and $400 \mathrm{mg} /$ $\mathrm{kg}$ ) significantly prevented the decrease in body weight. The dose of $400 \mathrm{mg} / \mathrm{kg}$ reduced the presence of diarrhea and rectal bleeding, although its comparison with the negative control only reached statistical significance on diarrhea. D-002 (25, 100 and $400 \mathrm{mg} / \mathrm{kg})$ significantly reduced the score of macroscopic lesions $(40.0 ; 43.3$ and $47.2 \%$ inhibition, respectively), the histological damage score (31.5; 53.7 and $67.1 \%$ inhibition, respectively) and the activity of MPO (73.2; 83.6 and $85.0 \%$ inhibition, respectively), compared to the negative control group. Sulfasalazine significantly reduced all variables studied. Conclusions. D-002 $(25,100$ and $400 \mathrm{mg} / \mathrm{kg})$ significantly protected the colonic mucosa in rats with severe inflammatory UC induced by DSS and ethanol.
\end{abstract}

Key words: Bees; Ulcerative colitis; Rats (Source: MeSH NLM).

\footnotetext{
Centro de Productos Naturales, Centro Nacional de Investigaciones Científicas, La Habana, Cuba

a Licenciada en Bioquímica; ${ }^{\text {b }}$ licenciada en Ciencias Farmacéuticas; ${ }^{\mathrm{c}}$ doctora en Medicina Veterinaria; ${ }^{\mathrm{d}}$ doctora en Ciencias Médicas; ${ }^{\text {e }}$ tecnólogo en Química Recibido: 30/08/2016 Aprobado: 22/02/2017 En línea: 28/06/2017
} 


\section{INTRODUCCIÓN}

La colitis ulcerativa (CU), que es una enfermedad inflamatoria crónica del intestino grueso (colon y recto), presenta una incidencia anual relativamente frecuente (8-12/100 000 personas), fundamentalmente en sujetos en edades comprendidas entre 15 y 40 años y entre 50 y 80 años ${ }^{(1,2)}$. Las principales manifestaciones clínicas de la CU, los cuales afectan la calidad de vida de los pacientes, incluyen la presencia de diarrea, sangrado en heces (melena), inflamación y dolor rectal ${ }^{(3)}$.

La etiología de la CU no ha sido del todo dilucidada, pero se conoce que se caracteriza por una notable infiltración de neutrófilos en las lesiones colónicas que se presentan con hiperemia, hemorragias y ulceraciones ${ }^{(4,5)}$. Los neutrófilos activados producen y liberan radicales hidroxilo $\left(\mathrm{OH}^{-}\right)$y aniones superóxido $\left(\mathrm{O}_{2}^{-}\right)$, los cuales causan daño tisular ${ }^{(6)}$. Otros mediadores inflamatorios (citoquinas y quimoquinas) también desempeñan un importante papel en el desarrollo y progresión de esta entidad patológica ${ }^{(7)}$.

La estrategia terapéutica para tratar la CU comprende el uso de medicamentos antiinflamatorios específicos como aminosalicilatos, corticosteroides y los anticuerpos que actúan sobre el factor de necrosis tumoral alfa (TNFa), capaces de controlar la expresión y/o neutralizar las funciones de los mediadores referidos anteriormente. Sin embargo, estos medicamentos pueden causar serios efectos adversos como agranulocitosis, infecciones severas, osteoporosis y tumores linfoides malignos ${ }^{(4,5)}$. Aunque es una enfermedad de etiología inflamatoria su terapéutica no incluye el uso de los antiinflamatorios no esteroideos (AINE) clásicos, debido a que la enfermedad se caracteriza por la presencia de ulceraciones colónicas (4) y estos agentes provocan lesiones en la mucosa gastrointestinal ${ }^{(8)}$. Por tales razones, se encuentra justificada la búsqueda de otras estrategias terapéuticas seguras y eficaces para el manejo de la CU.

El D-002 es una mezcla de seis alcoholes alifáticos primarios de alto peso molecular obtenida de la cera de abeja (Apis mellifera), cuyo componente principal es el triacontanol, seguido de hexacosanol, octacosanol, tetracosanol, dotriacontanol, y tetratriacontanol (9). Estudios experimentales y clínicos han demostrado que el D-002 presenta efectos antiinflamatorios, antioxidantes y gastroprotectores ${ }^{(10-17)}$.

Estudios experimentales previos, demostraron que el D-002, administrado profiláctica o terapéuticamente, redujo los cambios patológicos que caracterizan la $\mathrm{CU}$ inducida por ácido acético, en ratas, tan efectivamente como la sulfazalasina ${ }^{(18,19)}$ y resultó efectivo en el modelo de $\mathrm{CU}$ inducida por carragenina en cobayos ${ }^{(20)}$.

\section{MENSAJES CLAVE}

Motivación para realizar el estudio. La colitis ulcerativa (CU) es una enfermedad inflamatoria crónica que presenta una elevada incidencia en la población mundial, los medicamentos existentes no alcanzan la eficacia requerida y causan serios efectos adversos.

Principales hallazgos. La administración oral de D-002 (mezcla de alcoholes obtenida de la cera de abejas) protegió significativamente de la CU inducida por sulfato de dextrano y etanol en ratas, al reducir indicadores de sintomatología clínica.

Implicancias. El D-002 redujo los síntomas clínicos y las lesiones en tejido colónico en este modelo experimental. Esto podría representar un beneficio potencial para el mejoramiento de la calidad de vida y para el manejo de esta patología en seres humanos.

Vale destacar que tanto el modelo de CU inducida por ácido acético como por carragenina, son modelos de $\mathrm{CU}$ aguda ${ }^{(21)}$ y la eficacia del D-002 demostrada en ambos podría relacionarse con sus efectos antiinflamatorios y antioxidantes tras su administración aguda.

Por ello, era conveniente investigar los efectos del D-002 en un modelo de $\mathrm{CU}$, en el cual la inflamación intestinal se desarrolla como un proceso crónico, como el de la CU inducida por sulfato de dextrano (DSS, abreviaturas del nombre en inglés), el cual remeda en mayor medida la etiología de esta patología en el humano respecto a los modelos de CU aguda previamente estudiados. Sin embargo, su ejecución es muy costosa ${ }^{(22)}$.

Un modelo que reproduce las características de la $\mathrm{CU}$ humana, y resulta más económico, es el de la CU inducida por la combinación de bajas concentraciones de DSS y etanol. A pesar de estar clasificado como un modelo agudo, su ejecución reproduce una CU con inflamación severa que transcurre durante cinco días, lo que permite evaluar los tratamientos con esquemas de dosis repetidas. Además, este modelo garantiza un rápido desarrollo de ulceración e inflamación severa en la parte distal del colon de las ratas, reproduciendo características muy similares a las que se presentan en el humano como pérdida de peso, diarrea y hematoquecia ${ }^{(23)}$. Por tanto, el objetivo del presente trabajo consistió en investigar los efectos del D-002 sobre la CU inducida por DSS y etanol en ratas.

\section{MATERIALES Y MÉTODOS}

\section{ANIMALES}

Se utilizaron ratas (Ratus ratus) Sprague Dawley machos (200-250 g de peso corporal) provenientes del Centro Nacional para la Producción de Animales de Laboratorio, La Habana, Cuba, las cuales fueron adaptadas durante siete días a las condiciones de laboratorio (temperatura de 20 a 
$25^{\circ} \mathrm{C}$, humedad relativa de $60 \pm 5 \%$, ciclos de luz/oscuridad de 12 horas) con libre acceso al agua y la comida.

\section{ADMINISTRACIÓN Y DOSIFICACIÓN}

El D-002, (lote 030151211) obtenido en la Planta de Producción de Productos Naturales (CNIC, La Habana, Cuba) y la sulfazalacina (lote L $36 \mathrm{H} 0333$, Sigma-Aldrich, St. Louis, USA) se prepararon en forma de suspensión en vehículo Tween $20 / \mathrm{H} 2 \mathrm{O}(2 \%)$ y goma acacia/ $\mathrm{H} 2 \mathrm{O}$ (1\%), respectivamente.

Las ratas se distribuyeron aleatoriamente en seis grupos (10 ratas/grupo): un control cero que recibió vehículo por vía oral y agua intracolónica, y cinco a los que se les indujo la CU: un control negativo (vehículo), tres tratados con D-002 (25, 100 y $400 \mathrm{mg} / \mathrm{kg})$ y un control positivo con sulfazalacina $(200 \mathrm{mg} / \mathrm{kg}$ ) (sustancia de referencia). Todos los tratamientos (vehículo, D-002 y sulfazalacina) se administraron por vía oral mediante intubación intragástrica $(5 \mathrm{~mL} / \mathrm{kg})$ diariamente por cinco días.

Las dosis de D-002 se seleccionaron teniendo en cuenta que dosis entre 25 y $100 \mathrm{mg} / \mathrm{kg}$ han sido efectivas en los modelos de CU inducida por ácido acético o carragenina ${ }^{(18,20)}$, si bien decidimos ampliar el rango hasta $400 \mathrm{mg} / \mathrm{kg}$ dada la severidad inflamatoria del modelo empleado en este estudio. La dosis de sulfazalacina referida como eficaz en este modelo es $200 \mathrm{mg} / \mathrm{kg}^{(24)}$.

\section{DISEÑO EXPERIMENTAL}

Para la inducción de la CU, las ratas recibieron DSS (2\%) en el agua de tomar durante 3 días. Al cuarto día, se anestesió a las ratas con atmósfera de halotano y se les insertó un catéter por el recto mediante el cual se les instiló por el colon $0,5 \mathrm{~mL}$ de etanol al $30 \%$. Veinticuatro horas después (quinto día) las ratas se sacrificaron en atmósfera de halotano y se les extrajo el colon distal (8 $\mathrm{cm}$ de longitud) ${ }^{(23)}$.

\section{VARIABLES ANALIZADAS}

Determinación de indicadores de sintomatología clínica

En una balanza Sartorius se determinó la variación del peso corporal $(\triangle P C)$ calculando la diferencia entre el inicial (PCi), determinado antes de la DSS y el final (PCf), determinado antes del sacrificio. Se registró la presencia, o no, de diarrea y sangrado rectal de cada animal a las $24 \mathrm{~h}$ posteriores a la administración intracolónica de etanol.

Determinación del puntaje de daño macroscópico en la mucosa colónica

El colon distal previamente extraído se abrió longitudinalmente y se examinó bajo una lupa con aumento $3 X$. El daño visible se cuantificó mediante un puntaje de 0 a 5 , como sigue: 0 . Ningún daño; 1 . hiperemia localizada, sin ulceras ni erosiones; 2 . Úlceras o erosiones, sin inflamación; 3. Úlceras o erosiones con inflamación en un sitio; 4. Dos o más sitios de ulceración y/o inflamación, y 5 . Dos o más sitios mayores de inflamación y ulceración, o un sitio mayor de inflamación y ulceración con extensión de más de $1 \mathrm{~cm}{ }^{(25)}$.

\section{Determinación del puntaje de daño histológico en la} mucosa colónica

Para el análisis microscópico, las muestras de colon se fijaron en formaldehido tamponado al $10 \%$, se deshidrataron e incluyeron en parafina. Las secciones se colorearon con hematoxilina y eosina, y se examinaron utilizando un microscopio Carl Zeiss Primo Star. La evaluación del daño se realizó con el puntaje histológico descrito por Gaudio et al. ${ }^{(21)}$ y modificado por Chen Yan et al. ${ }^{(25)}$, como sigue:

(1) Destrucción del epitelio y glándulas: 0= morfología normal; 1= destrucción focal de la superficie epitelial y /o de las criptas; $2=$ destrucción zonal de la superficie epitelial y/o pérdida de criptas, y $3=u$ ulceración difusa de la mucosa y lo submucosa y /o pérdida de criptas;

(2) Dilatación de criptas glandulares: $0=$ aspecto normal; 1= dilatación focal; 2=dilatación zonal, y 3=dilatación difusa de criptas;

(3) Depleción y pérdida de células caliciformes: $0=$ aspecto normal; $1=$ ligera depleción de células caliciformes; $2=$ depleción moderada o zonal de células caliciformes, y 3 =depleción difusa o completa de células caliciformes;

(4) Infiltración de células inflamatorias: 0= ausencia de infiltración; 1=infiltrado en lámina propia o base de las criptas; $2=$ infiltración que alcanza la muscular de la mucosa, y 3=infiltración severa y extensa que alcanza submucosa y/o la capa muscular;

(5) Edema: 0=ausente; 1=focal; 2=zonal y/o moderadamente difuso, y $3=e x t e n s o$ y severo;

(6) Mucosa hemorrágica: $0=$ ausente; 1=focal; 2=zonal, y $3=$ difusa;

(7) Absceso en criptas: $0=$ =ausente; $1=$ focal; $2=$ zonal, y 3=difusa;

(8) Displasia: 0=ausente, 1=focal, 2=zonal, 3=difusa.

El puntaje de daño histológico en la mucosa colónica de cada animal se calculó como la sumatoria de los diferentes parámetros histológicos.

\section{Determinación de la actividad enzimática de la MPO}

Se tomaron alícuotas del tejido colónico que se homogenizaron en baño de hielo con homogenizador de cuchilla (Ultra-Turrax), en Buffer Tris- $\mathrm{HCl}$ (150 
$\mathrm{mmol} / \mathrm{L}$ y $\mathrm{pH} 7,4)$ y buffer fosfato $(50 \mathrm{mmol} / \mathrm{L}$ y $\mathrm{pH} 6$; conteniendo bromuro de hexadecil trimetilamonio al 0,5 $\%)$ para cuantificar la enzima MPO. Las muestras se almacenaron a $-20^{\circ} \mathrm{C}$ hasta su uso.

La actividad de la mieloperoxidasa (MPO) se determinó según la técnica del manual Worthington (26). Para ello, los homogenatos de tejido colónico se sonicaron por 10 segundos, y se congelaron $\left(-20^{\circ} \mathrm{C}\right)$ y descongelaron $\left(30^{\circ} \mathrm{C}\right)$ tres veces sucesivas. Al culminar la última descongelación, las muestras se centrifugaron a 12000 rpm por 25 min a 4 ${ }^{\circ} \mathrm{C}$ y se cuantificó la MPO en el sobrenadante. Para ello, se mezclaron $625 \mu \mathrm{L}$ de buffer fosfato (50 mmol/L, $\mathrm{pH}$ 6) (que contenía $0,167 \mathrm{mg} / \mathrm{mL}$ de dihidrocloruro de O-dianisidina), con $250 \mu \mathrm{L}$ de la muestra y $125 \mu \mathrm{L}$ de peróxido de hidrógeno $\left(\mathrm{H}_{2} \mathrm{O}_{2}\right)(0,0005 \%)$. Finalmente, en un espectrofotómetro se determinaron los cambios de absorbancia a $460 \mathrm{~nm}$ durante los 2 min siguientes a la reacción. La actividad de la MPO se refirió como unidad (U)/g de tejido.

Una U de MPO se definió como la degradación de un $\mu \mathrm{mol}$ de peróxido/min a $25^{\circ} \mathrm{C}$, la cual se determinó mediante la siguiente fórmula:

$$
U / g \text { de tejido }=\frac{\Delta \text { A min. } \times \text { Vol.cubeta }}{8,3 \times \text { Vol.muestra }} \times 10
$$

donde $\Delta A \min$. es el cambio de la absorbancia, Vol. cubeta es el volumen final de la cubeta y Vol. muestra es el volumen de muestra añadido $(\mu \mathrm{L})$.

\section{Determinación del porcentaje de inhibición}

El porcentaje de inhibición del efecto alcanzado por todos los tratamientos (D-002 y sulfazalacina) respecto al grupo control negativo se calculó como sigue:

$$
I(\%)=100-\frac{[(T-C c) \times 100]}{(C n-C c)}
$$

Donde $I(\%)$ es el porcentaje de inhibición, T es el valor medio del tratamiento; Cc es el valor medio del control cero y $\mathrm{Cn}$ es el valor medio del control negativo.

\section{ANÁLISIS ESTADÍSTICO}

La comparación de las variables continuas entre grupos se realizó con las pruebas no paramétricas de Kruskal Wallis y el de la $U$ de Mann Whitney para las comparaciones pares entre cada grupo y el control. Se utilizó el método de la probabilidad exacta de Fisher para la comparación entre grupos de variables categóricas (frecuencia de diarrea y sangrado rectal). A priori se estableció un nivel de $\alpha=0,05$ para la significación estadística. Los datos fueron procesados con el paquete de programas Statistic para Windows. (Release 4.2, Stat Soft, Inc USA). El estudio de relación dosis/efecto se realizó mediante el método de regresión lineal y correlación utilizando el programa Primer of Biostatistics (Stanton A, Glantz; copyright (c) 1992, McGraw-Hill, Inc Versión 3.01).

\section{RESULTADOS}

Los resultados de los efectos del D-002 sobre la variación de peso corporal y los síntomas clínicos en ratas con $\mathrm{CU}$ inducida por DSS y etanol se muestran en la Tabla 1. Como se aprecia, la inducción de la CU en los animales del grupo control negativo produjo una reducción significativa del PC con respecto al control cero, la cual fue atenuada de modo significativo y marcado por el tratamiento oral con D-002 (25 - 400 mg/kg) (61,8 -100\% de inhibición). El tratamiento oral con sulfazalacina $(200 \mathrm{mg} / \mathrm{kg}$ ) (sustancia de referencia) previno la reducción del PC de modo significativo y marcado (72,7\% de inhibición).

Como se observa, ningún animal del grupo control cero tuvo diarrea o sangrado rectal, síntomas manifiestos en un 77,8 y $33,3 \%$, respectivamente, en los animales del grupo control negativo. Las administraciones con D-002 (400 mg/kg) y sulfazalacina $(200 \mathrm{mg} / \mathrm{kg}$ ) redujeron significativamente el porcentaje de animales con diarrea (61,4 y $100 \%$ de inhibición, respectivamente) comparado al control negativo, al mismo tiempo que ningún animal tratado con D-002 (400 mg/kg) o sulfazalacina $(200 \mathrm{mg} / \mathrm{kg})$ presentó sangrado rectal (100\% de inhibición).

La Tabla 2 muestra los resultados de los efectos del D-002 sobre el puntaje de daño macroscópico e histológico en la mucosa colónica de ratas con CU inducida por DSS y etanol. El grupo control cero mostró puntajes cero, lo cual indica que no presentó daño macroscópico o histológico, mientras que esta variable se incrementó significativamente en el grupo control negativo. El tratamiento oral con D-002 (25, 100 y $400 \mathrm{mg} / \mathrm{kg})$ redujo significativamente, y dependiente de la dosis, el puntaje de las lesiones macroscópicas ( $p<0,05 ; r=0,036$ ) alcanzando porcentajes de inhibición de 40; 43,3 y $47,2 \%$, respectivamente, muy similar al efecto producido por la sulfazalacina $(200 \mathrm{mg} / \mathrm{kg})(43,3 \%)$.

Resultados coherentes se apreciaron con relación al daño histológico de la mucosa colónica, apreciándose que el D-002 (25, 100 y $400 \mathrm{mg} / \mathrm{kg}$ ) redujo significativamente el puntaje de daño histológico, alcanzando inhibiciones de 31,$5 ; 53,7$ y $67,1 \%$, respectivamente, un efecto no dependiente de la dosis. La administración oral con sulfazalacina $(200 \mathrm{mg} / \mathrm{kg})$ redujo de modo significativo $(65,1 \%$ de inhibición) el puntaje de daño histológico en mucosa colónica. 
Tabla 1. Efectos del D-002 sobre la variación del peso corporal y manifestaciones clínicas en ratas con colitis ulcerativa inducida por sulfato de dextrano y etanol

\begin{tabular}{lccccccc}
\hline Grupos & Dosis $(\mathbf{m g} / \mathbf{k g})$ & $\Delta \mathbf{P C}(\mathbf{g})$ & $\mathbf{I}(\%)$ & Diarreas (\%) & $\mathbf{I}(\%)$ & Sangrado rectal $(\%) \|$ & $\mathbf{I}(\%)$ \\
\hline Control cero & - & $20,5 \pm 3,7^{*}$ & - & $0 \S$ & - & 0 & - \\
Control negativo & - & $31,5 \pm 2,2^{*}$ & - & 77,8 & - & 33,3 & - \\
D-002 & 25 & $24,7 \pm 2,1^{*}$ & 61,8 & 55,5 & 28,6 & 22,2 & 33,3 \\
D-002 & 100 & $20,5 \pm 3,77^{*}$ & 100,0 & 55,5 & 28,6 & 22,2 & 33,3 \\
D-002 & 400 & $16,8 \pm 2,5^{\dagger}$ & 100,0 & $30^{\ddagger}$ & 61,4 & 0 & 100,0 \\
Control positivo Sulfazalacina & 200 & $23,5 \pm 3,1^{*}$ & 72,7 & $0 \S$ & 100,0 & 0 & 100,0 \\
\hline
\end{tabular}

${ }^{*} p<0,05 ;{ }^{\dagger} p<0,01$ comparado con el control negativo (prueba de la $U$ de Mann Whitney)

${ }_{\ddagger} \mathrm{p}<0,05 ;{ }^{\S} \mathrm{p}<0,01$ comparado con el control negativo (prueba de Fisher)

D-002: Mezcla de triacontanol, hexacosanol, octacosanol, tetracosanol, dotriacontanol, y tetratriacontanol (alcoholes alifáticos)

$\triangle \mathrm{PC}$ : Variación del peso corporal

" número de animales con el síntoma / total x 100

'(\%): porcentaje de inhibición

Los resultados referidos a la actividad de MPO en mucosa colónica de las ratas con $\mathrm{CU}$ se muestran en la Tabla 3. El grupo control negativo presentó un aumento significativo de la actividad de MPO con respecto al control cero. EI D-002 (25, 100 y $400 \mathrm{mg} / \mathrm{kg})$ redujo este aumento de modo significativo y marcado $(73,2 ; 83,6$ y $85,0 \%$, respectivamente), pero no dependiente de la dosis. A su vez, la sulfazalacina $(200 \mathrm{mg} / \mathrm{kg})$ redujo significativamente $(84,3 \%)$ la actividad de la MPO.

\section{DISCUSIÓN}

La administración oral de DSS e intracolónica de etanol, indujo $\mathrm{CU}$ en las ratas del grupo control negativo provocando síntomas clínicos (variación del peso corporal, presencia de diarrea y sangrado rectal), daño macroscópico y microscópico de la mucosa colónica así como incremento de la infiltración de neutrófilos (medido como actividad de MPO e histológicamente como un aspecto comprendido dentro del puntaje global de daño histológico), mientras que los animales del grupo control cero no presentaron síntomas clínicos, ni daño macroscópico o histológico y la actividad de MPO fue significativamente menor a la del grupo control negativo. Esto, unido a la eficacia de la sulfazalacina $(200 \mathrm{mg} / \mathrm{kg})$, sustancia de referencia, demuestra la validez de los presentes resultados en nuestras condiciones experimentales.

Aunque puede llamar la atención el hecho de que los animales del grupo control cero también presentaron una ligera reducción del peso corporal, vale señalar que esto responde al ayuno previo de 24 horas al que se sometieron todos los animales antes del sacrificio. En el caso de los animales del grupo control negativo, esta variación es mayor dado no solo el ayuno sino el desarrollo previo de la CU.

Los indicadores de CU medidos en el presente estudio se comportaron acorde a lo descrito por otros autores para el modelo aquí empleado y para el modelo tradicionalmente utilizado de CU inducida por DSS ${ }^{(25,27)}$ lo cual refuerza la validez de los presentes resultados.

La administración oral con D-002 (25, 100 y 400 mg/kg) a ratas protegió significativamente de la $\mathrm{CU}$ inducida por DSS y etanol, a todas las dosis ensayadas. Así, el D-002 redujo los indicadores de sintomatología clínica, el puntaje de lesiones macroscópicas e histológicas, así como el incremento de la actividad de MPO en colon.

Tabla 2. Efectos del D-002 sobre el puntaje de daño macroscópico e histológico en mucosa colónica de ratas con colitis ulcerativa inducida por sulfato de dextrano y etanol

\begin{tabular}{lccccc}
\hline Grupos & $\begin{array}{c}\text { Dosis } \\
(\mathbf{m g} / \mathbf{k g})\end{array}$ & $\begin{array}{c}\text { Puntaje daño } \\
\text { macroscópico }\end{array}$ & $\mathbf{I}(\%)$ & $\begin{array}{c}\text { Puntaje daño } \\
\text { histológico }\end{array}$ & $\mathbf{I}(\%)$ \\
\hline Control cero & - & $0 \pm 0^{\ddagger}$ & - & $0 \pm 0 \ddagger$ & - \\
Control negativo & & $4,62 \pm 0,18$ & - & $18,62 \pm 0,73$ & 31,5 \\
D-002 & 25 & $2,77 \pm 0,52^{*}$ & 40 & $12,75 \pm 0,55^{\ddagger}$ & 53,7 \\
D-002 & 100 & $2,62 \pm 0,70^{*}$ & 43,3 & $8,62 \pm 0,46 \ddagger$ & 67,1 \\
D-002 & 400 & $2,44 \pm 0,52^{\dagger}$ & 47,2 & $6,12 \pm 0,35^{\ddagger}$ & 65,1 \\
Control positivo Sulfazalacina & 200 & $2,62 \pm 0,26 \ddagger$ & 43,3 & $6,50 \pm 0,7^{\ddagger}$ & \\
\hline
\end{tabular}

${ }^{*} p<0,05 ; \dagger p<0,01 ; \ddagger p<0,001$ comparado con el control negativo (prueba de la $U$ de Mann Whitney)

D-002: Mezcla de triacontanol, hexacosanol, octacosanol, tetracosanol, dotriacontanol, y tetratriacontanol (alcoholes alifáticos)

$\mathrm{I}(\%)$ : porcentaje de inhibición 
Tabla 3. Efectos del D-002 sobre la actividad de MPO en mucosa colónica de ratas con colitis ulcerativa inducida por sulfato de dextrano y etanol

\begin{tabular}{lccc}
\hline Grupos & $\begin{array}{c}\text { Dosis } \\
(\mathbf{m g} / \mathbf{k g})\end{array}$ & $\begin{array}{c}\text { MPO } \\
\text { (U/g tejido) }\end{array}$ & $\mathbf{I}(\%)$ \\
\hline Control cero & - & $0,19 \pm 0,02^{*}$ & - \\
\hline Control negativo & - & $1,72 \pm 0,1$ & - \\
D-002 $_{25}$ & 25 & $0,60 \pm 0,06^{*}$ & 73,2 \\
D-002 $_{100}$ & 100 & $0,44 \pm 0,04^{*}$ & 83,6 \\
D-002 $_{200}$ & 400 & $0,42 \pm 0,04^{*}$ & 85,0 \\
Control positivo & 200 & $0,43 \pm 0,04^{*}$ & 84,3 \\
Sulfazalacina & & \\
\hline
\end{tabular}

* $p<0,001$ Comparado con el control negativo (prueba de la $U$ de Mann Whitney)

D-002: Mezcla de triacontanol, hexacosanol, octacosanol, tetracosanol, dotriacontanol, y tetratriacontanol (alcoholes alifáticos)

MPO: Mieloperoxidasa

I (\%): porcentaje de inhibición

Por tanto, este estudio constituye el primer reporte de los efectos beneficiosos del D-002 en este modelo de $\mathrm{CU}$, lo cual concuerda con los resultados obtenidos en los modelos de CU aguda reportados previamente ${ }^{(18,19)}$.

Teniendo en cuenta que la CU es una enfermedad que afecta la calidad de vida de los pacientes, dado sus molestos síntomas (presencia de diarrea, sangrado en las heces, inflamación, dolor rectal y pérdida de peso corporal entre otros), ${ }^{(3,28)}$ el hecho de que el D-002 redujera los síntomas clínicos relacionados con el desarrollo de la $\mathrm{CU}$ en este modelo de gran similitud con la etiología de la enfermedad en el humano, representa un beneficio potencial para el alivio de los síntomas en pacientes con $\mathrm{CU}$, si bien tal resultado deberá ser corroborado por ulteriores estudios clínicos.

Los efectos protectores del D-002 (25, 100 y 400 mg/kg) sobre el daño en mucosa colónica, evaluado macroscópica e histológicamente, sustentan los resultados obtenidos sobre la sintomatología clínica. Así, se observó una correspondencia entre los porcentajes de inhibición marcados alcanzados por el D-002 sobre el daño histológico en mucosa colónica $(67,1 \%)$ y las diarreas $(61,4 \%)$, importante síntoma clínico de la CU, si bien la reducción del puntaje de daño macroscópico fue moderada (47,2\% de inhibición)

Además, la mayor eficacia del D-002 (25, 100 y 400 mg/kg) sobre la sintomatología clínica, los daños macroscópico e histológico y la actividad de MPO se logró con la mayor dosis ensayada de $400 \mathrm{mg} / \mathrm{kg}$, y resultó efectivo a partir de la menor dosis ensayada de $25 \mathrm{mg} / \mathrm{kg}$. Dado que no se evaluaron dosis superiores a $400 \mathrm{mg} / \mathrm{kg}$ no se alcanzó el efecto máximo, por lo que no podemos descartar que con dosis superiores se alcancen mayores eficacias, como tampoco evaluamos dosis inferiores a $25 \mathrm{mg} / \mathrm{kg}$ por lo que no pudimos caracterizar la dosis mínima efectiva.
Por tanto, estudios ulteriores deberán evaluar un rango de dosis aun más amplio en aras de profundizar en el estudio de la relación dosis-efecto en este modelo de $\mathrm{CU}$, de modo que permita obtener la dosis mínima efectiva y la dosis efectiva máxima.

Por otra parte, el hecho de que la actividad de la MPO en tejido colónico del grupo control negativo resultara significativamente superior a la del control cero, constituye una evidencia más del papel que desempeña la inflamación, específicamente la infiltración de neutrófilos, en el desarrollo de la CU como enfermedad inflamatoria. Aquí, la inhibición marcada del D-002 sobre la actividad de la enzima (85\%) sugiere que la protección del D-002 sobre la CU está asociada a una inhibición o modulación de la infiltración de neutrófilos al tejido dañado, lo cual se pudo constatar en la evaluación de la infiltración de células inflamatorias comprendida en la evaluación histológica.

Estudios previos han demostrado que el mecanismo antiinflamatorio del D-002 involucra la inhibición dual de las enzimas 5-lipooxigenasa y ciclooxigenasa tipo II (29). La inhibición de la primera conduce a una inhibición en la formación del leucotrieno B4 (LT B4), potente agente quimiotáctico de neutrófilos, por lo que la eficacia del D-002 para reducir la actividad de MPO aquí observada pudiera estar asociada a la inhibición de la 5-LOX. Por tanto, estos resultados concuerdan con la eficacia del D-002 para reducir la generación del LT B4 demostrada por estudios previos ${ }^{(10)}$ y con la eficacia del D-002 para reducir la actividad de la MPO demostrada en otros modelos de inflamación ${ }^{(30)}$.

Los resultados del presente estudio sugieren que la protección que ejerce elD-002 sobre el colon en el modelo de CU inducida por DSS y etanol en ratas, pudiera estar asociada a su marcada eficacia antiinflamatoria, dada por la reducción de la actividad de MPO como marcador de infiltración de neutrófilos.

Por otra parte, dado el relevante papel de los radicales libres en la etiología de la CU (6) y que diferentes sustancias con propiedades antioxidantes han resultado efectivas en modelos experimentales de CU ${ }^{(27,31,32)}$, es lógico suponer que los efectos antioxidantes del D-002 estén involucrados en su eficacia en este modelo, lo cual deberá ser dilucidado por ulteriores estudios.

En conclusión, el tratamiento oral con D-002 (25, 100 y $400 \mathrm{mg} / \mathrm{kg}$ ) redujo significativamente la sintomatología clínica y el puntaje de daño macroscópico e histológico en mucosa colónica de ratas con CU inducida por DSS y etanol, efecto que estuvo asociado a su actividad antiinflamatoria al reducir la actividad de la MPO en colon.

Contribución de autoría: VMC participó en la concepción del trabajo, ejecución experimental, recolección de datos, redacción del artículo, y aprobación final. YRC y ZZR participaron en 
la ejecución experimental, obtención y análisis de datos, y aprobación final. MNP y MVC participaron en el análisis histológico y aprobación final. YPG, AOY y SJD participaron en las determinaciones bioquímicas y aprobación final. RMF participó en la concepción del trabajo y lo asesoró.
Fuentes de financiamiento: Centro Nacional de Investigaciones Científicas (CENIC) de Cuba.

Conflictos de interés: los autores declaran no tener conflictos de interés.

\section{REFERENCIAS BIBLIOGRÁFICAS}

1. Kornbluth A, Sachar DB. Ulcerative colitis practice guidelines in adults (update): American College Gastroenterology Practice Parameters Committee. Am J Gastroenterol. 2010;105(3):501-23. doi:10.1038/ ajg.2009.727

2. Burisch J, Munkholm P. The epidemiology of inflammatory bowel disease. Scand J Gastroenterol 2015; $50(8): 942-51$. doi: 10.3109/00365521.2015.1014407.

3. Di Sabatino A, Biancheri P, Rovedatti L, Macdonald TT, Corazza GR. Recent advances in understanding ulcerative colitis. Intern Emerg Med. 2012; 7:10311. doi: $10.1007 / \mathrm{s} 11739-011-0719-\mathrm{z}$

4. Fries W, Comunale S. Ulcerative colitis: pathogenesis. Curr Drug Targets. 2011; 12:1373-82.

5. Xavier RJ, Podolsky DK. Unravelling the pathogenesis of inflammatory bowel disease. Nature. 2007; 448(7152): 427-34.

6. Ishihara T, Tanaka K, Tasaka Y, Namba T, Suzuki J, Ishihara T, et al. Therapeutic effect of lecithinized superoxide dismutase against colitis. J Pharmacol Exp Ther. 2009, 328(1):152-64. doi: 10.1124/ jpet.108.144451.

7. Biesiada G, Czepiel J, Ptak-Belowska A, Targosz A, Krzysiek-Maczka G, Strzalka M, et al. Expression and release of leptin and proinflammatory cytokines in patients with ulcerative colitis and infectious diarrhea. J Physiol Pharmacol. 2012; 63:471-81.

8. Takeuchi K, Tanaka A, Kato S, Amagase $\mathrm{K}$, Satoh H. Roles of COX inhibition in pathogenesis of NSAID-induced small intestinal damage. Clin Chim Acta. 2010; 411:459-466. doi: 10.1016/j. cca.2009.12.026.

9. Mas R. D-002: A product obtained from beeswax. Drugs of the Future 2001; 26:731-744

10. Carbajal D, Molina V, Valdes S, Arruzazabala ML, Mas R, Magraner J. Anti-inflammmatory activity of D-002: An active product isolated from beeswax. Prostaglandins Leukot Essent Fatty Acids. 1998;59(4): 235-8.

11. Carbajal D, Molina V, Valdés $S$, Arruzazabala ML, Mas R. Anti-ulcer activity of higher primary alcohols of beeswax. J Pharm Pharmacol. 1995;47(9):731-3.

12. Carbajal D, Molina V, Valdés $S$, Arruzazabala ML, Rodeiro I, Mas R, et al. Possible cytoprotective mechanism in rats of D-002 an anti-ulcerogenic product isolated from beeswax. J Pharm Pharmacol. 1996;48(8):858-60.

13. Carbajal D, Molina V, Noa M, Valdes S, Arruzazabala ML, Aguilar A, et al. Effects of D-002 on gastric mucus composition in ethanol induced ulcer. Pharmacol Res. 2000;42:329-32.

14. Menéndez R, Amor AM, González RM, Jiménez S, Mas R. Inhibition of rat microsomal lipid peroxidation by the oral administration of D-002. Braz J Med Biol Res. 2000;33:85-90.

15. Fernández L, Terry $\mathrm{H}$, Quiñones AM, Díaz B, Hernández ML, IIlnait J, et al. Effects of Abexol in |middle-aged and older subjects: an open follow-up. Rev CENIC Cien Biol. 2008;39(1):3-8

16. Illnait J, Terry $H$, Mas $R$, Fernandez L, Carbajal D. Effects of D-002, a product isolated from beeswax, on gastric symptoms of patients with osteoarthritis treated with piroxicam: a pilot study. J Med Food. 2005;8(1):63-8. doi: 10.1089 /jmf.2005.8.63

17. Hano O, Illnait J, Mas R, Fernández L Piñol F, Fernández J. Effects of D-002, a Product Isolated from Beeswax, on Duodenal Ulcer: A Double-Blind, Placebo-Controlled Study. Curr Ther Res. 2001;62:394-407.

18. Noa M, Más R, Carbajal D, Valdés $S$. Effect of D-002 on acetic acid-induced colitis in rats at single and repeated doses. Pharmacol Res. 2000;41(4):391-5. doi: 10.1006/phrs. 1999.0596

19. Noa M, Carbajal D, Molina V, Valdés S, Más R. Comparative study of D-002 versus sulfasalazine on acetic acid-induced colitis in rats. Drugs Exp Clin Res. 2000;26(1):13-17.

20. Noa M, Mas R. Effect of D-002 on the preulcerative phase of carrageenan-induced colonic ulceration in the guinea-pig. J Pharm Pharmacol. 1998; 50(5):549-53.

21. Elson CO, Sartor RB, Tennyson GS Riddell RH. Experimental models of inflammatory bowel disease. Gastroenterology. 1995;109(4):1344-67.

22. Gaudio E, Taddei G, Vetuschi A, Sferra R, Frieri G, Ricciardi G. Dextran sulfate sodium (DSS) colitis in rats: clinical, structural, and ultrastructural aspects. Dig Dis Sci. 1999;44:1458-75.

23. Chen Y, Si J, Liu W, Cai J, Du Q, Wang $\mathrm{L}$, et al. Induction of experimental acute ulcerative colitis in rats by administration of dextran sulfate sodium at low concentration followed by intracolonic administration of $30 \%$ ethanol. J Zhejiang Univ Sci B. 2007; 8(9):632-7.
24. Xing JF, Sun JN, Sun JY, Hu SS, Guo ChN, Wang ML, et al. Protective effect of shikimic acid on acetic acid induced colitis in rats. J Med Plants Res. 2012;6:2011-8.

25. Chen Y, Si JM, Liu WL, Cai JT, $\mathrm{Du} \mathrm{Q}$, Wang LJ, et al. Induction of experimental acute ulcerative colitis in rats by administration of dextran sulfate sodium at low concentration followed by intracolonic administration of $30 \%$ ethanol. J Zhejiang Univ Sci B. 2007; 8(9):632-7. doi: 10.1631/jzus.2007. B0632

26. Worthington Enzyme Manual. Freehold, New Jersey: Worthington Biochemical Corp;1972. p.43-5.

27. Vasina V, Broccoli M, Ursino MG, Canistro D, Valgimigli L, Soleti A, et al. Non-peptidyl low molecular weight radical scavenger IAC attenuates DSS-induced colitis in rats. World J Gastroenterol. 2010;16(29):3642-50.

28. Danese S, Fiocchi C. Ulcerative Colitis. N England J Med. 2011;365(18):1713-25.

29. Pérez Y, Oyarzábal A, Ravelo Y, Mas $\mathrm{R}$, Jiménez $\mathrm{S}$, Molina V. Inhibition of Ciclooxigenase and 5-Lipooxigenase enzymes by D-002 (beeswax alcohols). Curr Top Nutr Res. 2014;12(1/2):13-8.

30. Ravelo Y, Molina V, Carbajal D, Arruzazabala ML, Mas R, Oyarzabal $A$, et al. Effects of single oral and topical administration of D-002 (beeswax alcohols) on xylene induced ear edema in mice. Lat Am j pharm. 2010;29(8):1451-4.

31. Cheah KY, Bastian SE, Acott TM, Abimosleh SM, Lymn KA, Howarth GS. Grape Seed Extract Reduces the Severity of Selected Disease Markers in the Proximal Colon of Dextran Sulphate Sodium-Induced Colitis in Rats. Dig Dis Sci. 2013;58(4):970-7. doi: 10.1007/ s10620-012-2464-1.

32. Grimstad T, Bjørndal B, Cacabelos D, Aasprong OG, Janssen EA, Omdal $\mathrm{R}$, et al. Dietary supplementation of krill oil attenuates inflammation and oxidative stress in experimental ulcerative colitis in rats. Scand J Gastroenterol. 2012;47(1):49-58. doi: $10.3109 / 00365521.2011 .634025$.

Correspondencia: Vivian Molina Cuevas Dirección: Calle 198 el 19 y 21 Atabey, Playa, La Habana. Cuba Teléfono: (+053)2714225

Correo electrónico:vivian.molina@cnic.edu.cu 